\title{
Suscetibilidade de dois tipos de solo à compactação
}

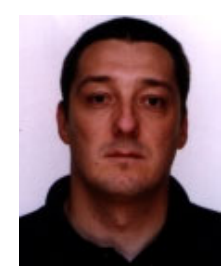

Pedro H. Weirich Neto ${ }^{1}$, André L. T. da Rosa² \& Jaime A. Gomes ${ }^{1}$

\footnotetext{
1 Laboratório de Mecanização Agrícola (Lama), Universidade Estadual de Ponta Grossa (UEPG). Praça Santos Andrade s/n, CEP 84010-330, Ponta Grossa, PR. Fone: (42) 220-3092. E-mail: lama1@uepg.br (Foto)

2 Sementes Pantera, Alto Taquari - MT
}

Protocolo $128-16 / 11 / 1999$

\begin{abstract}
Resumo: Com vistas ao aumento da produção agrícola, optou-se no Brasil, pelo acréscimo de área cultivada; para tal o uso indiscriminado da motomecanização mostrou-se eficiente em um primeiro momento, porém acarretando problemas à física do solo no decorrer dos anos. Paralelamente, práticas conservacionistas se fazem presentes, vide semeadura sob a palha (Plantio Direto), uma realidade no sul do Brasil. Sendo assim, avaliou-se a suscetibilidade de compactação de dois tipos de solo, ambos com sistema de semeadura sob a palha. Para dimensionar a suscetibilidade a compactação, utilizou-se ensaio de Proctor. Os solos foram ensaiados com e sem a presença de matéria orgânica livre. Os resultados demonstraram que para o Cambissolo, textura média, não houve diferença nos valores que representam a suscetibilidade, enquanto no Latossolo Vermelho, textura argilosa, não houve diferença entre a máxima densidade aparente, porém houve diferença significativa no conteúdo de água para a máxima densidade, o mesmo alterou-se de $0,249 \mathrm{~g} \mathrm{~g}^{-1}$ no ponto de máxima compactação no ensaio sem a presença de matéria orgânica livre, para 0,283 $\mathrm{g} \mathrm{g}^{-1}$ no ponto de máxima compactação, quando da presença da matéria orgânica livre.
\end{abstract}

Palavras-chave: ensaio de Proctor, plantio direto, matéria orgânica

\section{Compaction susceptibility of two classes of soil}

\begin{abstract}
For increasing agricultural production, Brazil opted to enlarge the cultivated area, and indiscriminate mechanization was efficient at the first moment, although it altered adversely the soil properties with the passage of time. At the same time, conservation practices, like the direct sowing under the straw (No Tillage) were adopted which is a reality in Southern Brazil. Considering this, the susceptibility for compaction of two classes of soil was evaluated under no tillage system, with and without free organic matter. The Proctor test was used to measure the degree of compaction. The results of a medium texture Cambissol showed there was no difference in susceptibility for compaction. The clay textured Red Latossol, showed no difference in the maximum apparent density, although there was significant difference in water content at maximum density. The values altered from $0.249 \mathrm{~g} \mathrm{~g}^{-1}$, at a maximum compaction point without free organic matter to $0.283 \mathrm{~g} \mathrm{~g}^{-1}$ with free organic matter.
\end{abstract}

Key words: Proctor, no tillage, organic matter

\section{INTRODUÇÃO}

Com a abertura de novas fronteiras agrícolas e a tecnificação da agricultura, o uso indiscriminado de máquinas e implementos agrícolas passou a ser uma constante, provocando mudanças prejudiciais às propriedades físicas dos solos, já relatadas por (Primavesi, 1986). Uma dessas mudanças seria o aumento da densidade do solo, em conseqüência da redução do seu volume; este processo é regido por inúmeras variáveis, como interação entre propriedades do solo, fatores ambientais e o tipo de manejo agrícola utilizado (Jorge, 1993).
Quanto às propriedades do solo e aos fatores ambientais, deve-se ter o aprendizado da convivência, porém quanto ao tipo de manejo, soluções têm sido apresentadas, em que um exemplo é a semeadura sob a palha (plantio direto), onde o mínimo de revolvimento do solo é desejado, diminuindo o número de operações agrícolas e, conseqüentemente, o tráfego de máquinas e implementos. Um dos pilares desse sistema é a rotação de culturas, muitas vezes abrindo-se mão da exportação de grãos, com vistas à manutenção da cobertura vegetal do solo, o que veio transformar vários processos químicos, físicos e biológicos. 
Objetivando-se o levantamento de dados que gerem recomendações quanto à melhor forma de interação máquina/ solo, vários estudos foram realizados em campo, sempre com o uso de metodologias complexas.

O segmento de pesquisa em máquinas agrícolas testa dispositivos de interação máquina/solo (Lanças \& Upadhyaya, 1997; Neujahr et al., 1998; Schlosser et al., 1998 e Trein \& Bergamaschi, 1998); enquanto o segmento de pesquisa em ciência do solo trabalha na interação de variáveis, trabalhando com modelagem matemática (Kondo \& Dias Jr., 1998). A procura por metodologias aplicáveis em laboratórios é cada vez mais comum, pois além do controle mais eficaz de certas variáveis, a repetibilidade é garantida. Outras áreas da ciência realizam o ensaio de Proctor Normal, como o caso típico de estradas de rodagem, em que a máxima compactação é perseguida através do ponto de conteúdo de água ótimo (Martins Jr., 1964).

Alguns pesquisadores da área agrícola já utilizaram o ensaio de Proctor; Novak et al. (1992) praticamente validaram a metodologia; e Figueiredo et al. (1998) compararam sistemas de manejo de solo através dele.

O conteúdo de água de um solo é considerado, por alguns autores, como um dos fatores mais importantes quando do manejo agrícola; além de influir nas propriedades físicas do solo quando da interação com o processo agrícola (Novak et al., 1992 e Camargo \& Alleoni, 1997), e em alguns processos químicos, bioquímicos e biológicos do solo, o que o torna importante no fenômeno de compactação (Forsythe, 1980).

Outro fator de grande importância, de variação temporal menos intensa, é a matéria orgânica presente no solo. O uso de coberturas ou fertilizantes que venham promover a presença e o aumento de matéria orgânica no solo é um importante aliado contra o processo de compactação (Buckman, 1976; Jorge, 1985; Kiehl, 1985; Ekwue \& Stone, 1995 e Camargo \& Alleoni, 1997).

A matéria orgânica pode ser classificada de duas formas: uma constituída de restos vegetais e de animais, em início de decomposição, não ligados à fração mineral, neste trabalho denominada matéria orgânica livre (mol); e outra intimamente ligada aos componentes minerais. Interessante considerar-se, ainda, como diferencial, tanto o aspecto quantitativo como o qualitativo da matéria orgânica presente no solo (Lassus, 1990).

Além dos solos com teores mais elevados de matéria orgânica apresentarem densidades aparentes menores, estes possuem maior capacidade de retenção de água (Kiehl, 1985 e Salton \& Mielniczuk, 1995), sendo a capacidade de retenção proporcional à presença de matéria orgânica livre (Kiehl, 1985).

Assim, realizou-se um trabalho em dois solos comumente encontrados, objetivando-se a curva de ajuste, conforme metodologia do ensaio de Proctor (Martins Jr., 1964), com e sem a presença de matéria orgânica livre.

\section{MATERIAL E MÉTODOS}

Os ensaios foram realizados no Laboratório de Mecanização Agrícola - LAMA, do Departamento de Ciência do Solo e Engenharia Agrícola - Desolo, Campus "Uvaranas" da Universidade Estadual de Ponta Grossa - UEPG, Paraná, Brasil.

As amostras avaliadas provinham da Fazenda Escola "Capão da Onça", esses solos foram um Cambissolo e um Latossolo Vermelho, caracterizados conforme Tabela 1. As amostras foram compostas de solo e os resíduos vegetais presentes a uma profundidade de $0-200 \mathrm{~mm}$, desprezando-se os restos culturais da superfície.

Os tipos de solos escolhidos para este trabalho constituem dois dos mais importantes, pois ocupam grande parte do território brasileiro. Os Latossolos apresentam grande variação textural e disparidades quanto à infiltração e capacidade de retenção de água, além de serem profundos em terrenos planos e suavemente ondulados, fatores determinantes quanto ao uso agrícola (Oliveira, 1992). Os Cambissolos se caracterizam por estarem em desenvolvimento, de profundidade moderada e não apresentam restrições de drenagem, tornando-se de bom potencial agrícola (Oliveira, 1992).

Os dois solos estudados estavam sob manejo de semeadura sob a palha, e os dados relativos à rotação de culturas são apresentados na Tabela 2. O início do cultivo agrícola do Cambissolo foi semeadura sob a palha (Plantio Direto). Na safra agrícola de 95/96, o sistema de semeadura utilizado no Latossolo Vermelho era o preparo convencional (PC).

Os solos foram submetidos à metodologia denominada Proctor, descrita por Martins Jr. (1964) e utilizada como análise de rotina pelo Departamento Nacional de Estradas de Rodagem - DNER. Em um dos tratamentos, antes da realização do ensaio de Proctor, a matéria orgânica livre foi retirada por catação manual durante o processo de secagem do solo ao ar.

Considerou-se cada classe de solo estudada como um experimento individual, analisando como variável independente à presença ou não de matéria orgânica livre e, como variáveis dependentes, o ponto de densidade aparente máxima, o conteúdo de água onde se dava a densidade aparente máxima e a área sob a curva de regressão que representava o fenômeno. Todas as variáveis dependentes foram calculadas, levando-se em consideração a curva ajustada aos valores obtidos pelo ensaio de Proctor.

Utilizou-se delineamento inteiramente casualizado, com três repetições; as médias foram comparadas pelo teste de Tukey, através do programa computacional denominado Estat. (V. 2.0) da Universidade Estadual Paulista - Campus de Jaboticabal.

\section{RESULTADOS E DISCUSSÃO}

\section{Cambissolo}

Nos ensaios correspondentes ao Cambissolo a curva polinomial de segundo grau foi a que melhor se adaptou ao fenômeno (Fig. 1).

Tabela 1. Característica dos solos utilizados

\begin{tabular}{|c|c|c|c|c|c|c|}
\hline \multirow{2}{*}{ Solo } & \multirow{2}{*}{$\begin{array}{c}\mathrm{DA}^{*} \\
\mathrm{~kg} \mathrm{dm}^{-3}\end{array}$} & \multirow{2}{*}{$\begin{array}{l}\text { Carbono } \\
\mathrm{g} \mathrm{kg}^{-1}\end{array}$} & \multirow{2}{*}{$\begin{array}{l}\text { Conteúdo de Água } \\
\qquad \mathrm{g} \mathrm{g}^{-1}\end{array}$} & Areia & Silte & Argila \\
\hline & & & & \multicolumn{3}{|c|}{$\mathrm{g} \mathrm{kg}^{-1}$} \\
\hline Latossolo & 1,510 & 29 & 0,3909 & 362 & 138 & 500 \\
\hline Cambissolo & 1,700 & 17 & 0,1631 & 790 & 70 & 140 \\
\hline
\end{tabular}

* DA - Densidade aparente no momento da coleta, determinada pelo método do anel volumétrico (Kiehl, 1979)

** Conteúdo de água médio, profundidade de 0-200 mm, no momento da coleta das amostras, 13 de março de 1998; precipitação pluviométrica no período de 1 a 12 de março de 1998 foi de 196 mm 
Tabela 2. Manejo de rotação de culturas dos solos estudados

\begin{tabular}{lcccccc} 
& \multicolumn{5}{c}{ Ano } & \\
\cline { 2 - 6 } & $95 / 96$ & $96 / 96$ & $96 / 97$ & $97 / 97$ & $97 / 98$ & $98 / 98$ \\
\hline $\begin{array}{l}\text { A. Cambissolo } \\
\text { Cultura }\end{array}$ & Campo nativo & Aveia preta & Soja & Aveia preta & Soja & Aveia preta \\
$\begin{array}{l}\text { B. Latossolo } \\
\text { Cultura }\end{array}$ & Soja (PC) & Cevada & Milho & Aveia preta & Soja & Cevada \\
\hline
\end{tabular}
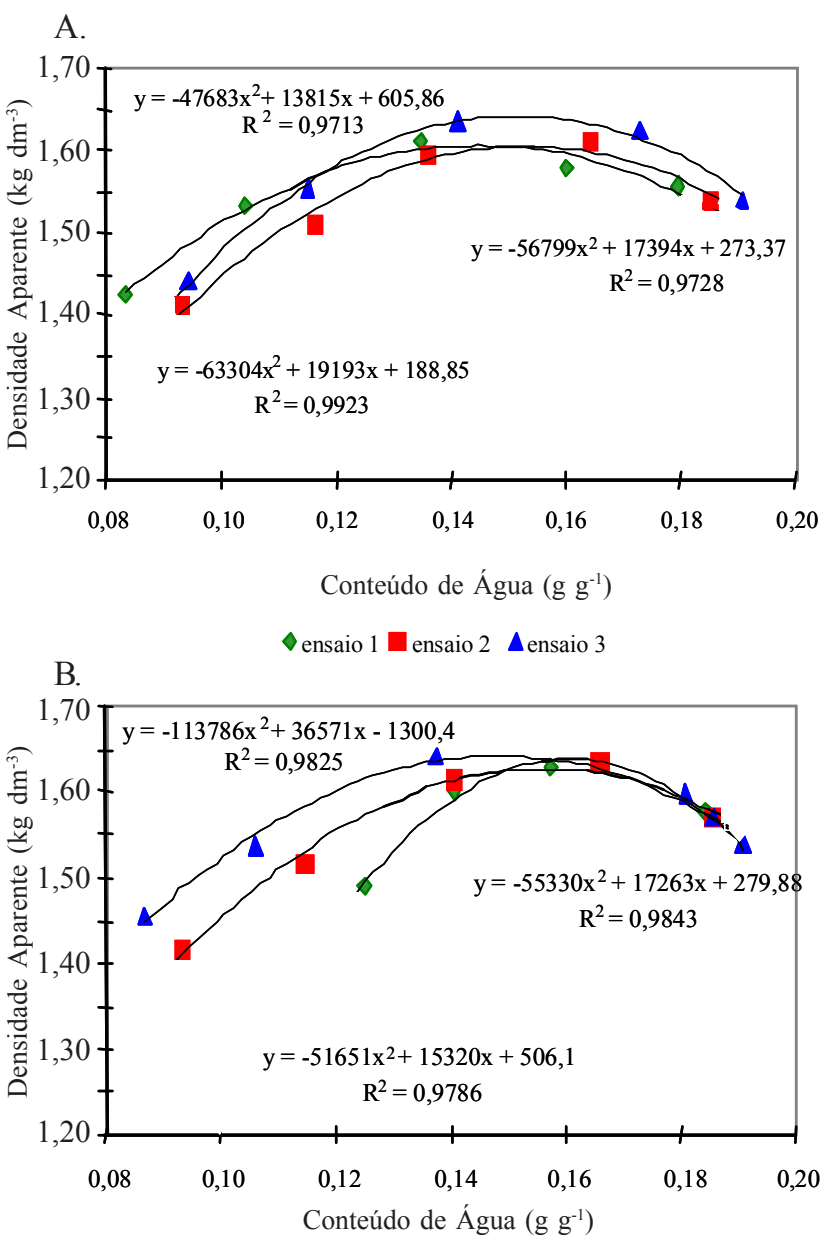

$\diamond$ ensaio 1(m.o.l.=13,92g) Шensaio 2 (m.o.1.=13,91g) $\boldsymbol{\Lambda}$ ensaio 3 (m.o.1.=19,55g)

Figura 1. Curvas de regressão a partir de determinações do ensaio de Proctor para um Cambissolo textura média (A) sem e (B) com a presença de matéria orgânica livre (m.o.l) visual

Observa-se, conforme Figura 1A, que o conteúdo de água onde houve a máxima densidade aparente, para o ensaio sem a presença de matéria orgânica livre, variou de 0,1449 a $0,1531 \mathrm{~g} \mathrm{~g}^{-1}$, com valores máximos de densidade aparente variando de 1,605 a $1,643 \mathrm{~kg} \mathrm{dm}^{-3}$, valores esses condizentes com a textura do solo estudado.

Para os ensaios do solo com a presença de matéria orgânica livre (Fig. 1B), os valores de conteúdo de água em que ocorreu máxima densidade aparente variaram de 0,1493 a $0,1609 \mathrm{~g} \mathrm{~g}^{-1}$, enquanto os valores de densidade aparente foram de 1,626 a $1,641 \mathrm{~kg} \mathrm{dm}^{-3}$, com conteúdo de matéria orgânica livre seca visualmente identificada, variando de 13,91 a 19,55 g.

A variável densidade aparente do solo apresentou comportamento respeitando uma equação de segundo grau conforme incremento do conteúdo de água para uma mesma solicitação mecânica externa. Convém lembrar que em conteúdos de água do solo mais baixos, existe por parte do material mineral do solo, elevado atrito interno que não permite um melhor arranjo e conforme se adiciona água, esta age como agente lubrificante, melhorando o arranjo entre as partículas, e aumentando a densidade aparente, porém, quando chega a determinado conteúdo, a água começa a ocupar os espaços e, sendo um fluido, por isso incompressível, com uma mesma solicitação mecânica externa, este solo chega a densidades menores.

A análise das variáveis dependentes é mostrada na Tabela 3A. Tal análise não se mostrou significativamente diferente, fato que se explica pelo tipo de solo estudado, o qual, mesmo de textura média, apresenta partículas maiores em relação a outros solos, cujo efeito da presença de matéria orgânica livre não apresenta grande variação no seu comportamento em relação à suscetibilidade à compactação. Interessante ressaltar que os valores elevados de matéria orgânica livre são explicados, talvez, pelo manejo do solo, tendo em vista que nesta área nunca houve a prática do sistema convencional de preparo do solo.

Tabela 3. Análise estatística das médias dos valores de conteúdo de água* onde a densidade é máxima, densidade aparente máxima e área sob a curva de ajuste, conforme ensaio de Proctor para (A) Cambissolo textura média e (B) Latossolo Vermelho Escuro, textura argilosa

\begin{tabular}{cccc} 
& Conteúdo de & Densidade & Área sob a \\
Condições & Densidade & Aparente & Curva de \\
& Aparente & Máxima & Ajuste \\
& Máxima $\left(\mathrm{g} \mathrm{g}^{-1}\right)$ & $\left(\mathrm{kg} \mathrm{dm}^{-3}\right)$ & $\left(\mathrm{g} \mathrm{dm}^{-3}\right)^{* *}$ \\
\hline
\end{tabular}

A. Cambissolo textura média

Amostras

sem m.o.l

Amostras

com m.o.l.

0,1498 a

$1,618 \mathrm{~A}$

80,19 a

0,1553 a

$1,635 \mathrm{~A}$

80,43 a

B. Latossolo Vermelho Escuro, textura argilosa

Amostras sem m.o.l.

$0,2486 \mathrm{a}$

1,356 a

119,36 a

Amostras

com m.o.l.

$0,2805 \mathrm{~b}$

$1,316 \mathrm{a}$

116,50

* Médias seguidas pelas letras iguais não diferem estatisticamente em nível de 5\%, Tukey ** Intervalo de integração, conteúdo de água de 0,120 a $0,170 \mathrm{~g} \mathrm{~g}^{-1}$

Observa-se que, com um valor baixo de conteúdo de água, o solo estudado alcança o valor máximo de densidade, devido ao tamanho das partículas de solo, de grande área específica. Os valores elevados da densidade aparente se justificam, em parte pelo tamanho das partículas do solo estudado e devido ao ensaio de Proctor, que superestima a carga mecânica aplicada. 
Para o cálculo da área sob a curva de compactação utilizouse o intervalo de 0,120 a $0,170 \mathrm{~g} \mathrm{~g}^{-1}$ para a integração das curvas, conforme ajuste dos valores obtidos pelo ensaio de Proctor, que eram comuns às amostras estudadas. Neste item seria interessante um estudo de campo para determinação de valores de conteúdo de água reais e com maior probabilidade de ocorrência; de posse desses valores, os mesmos seriam os intervalos para a integração da curva de compactação, chegando-se a valores de área que representariam mais fielmente a suscetibilidade de tal solo à compactação.

\section{Latossolo}

Nos ensaios correspondentes ao Latossolo a curva polinomial de segundo grau foi a que melhor se adaptou ao fenômeno (Fig. 2).
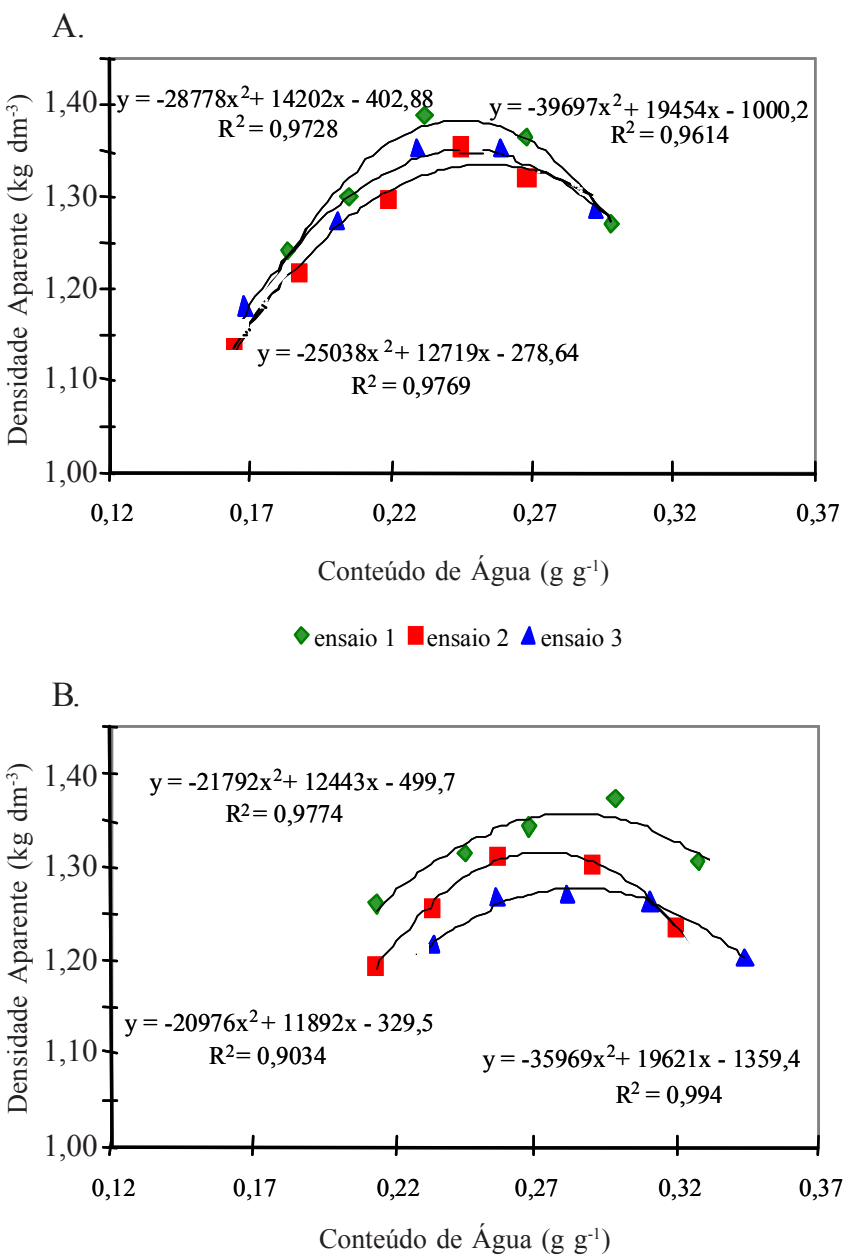

$\checkmark$ ensaio 1 (m.o.l.=2,78g)

Figura 2. Curvas de regressão obtidas a partir de determinações do ensaio de Proctor para um Latossolo Vermelho textura argilosa (A) sem e (B) com a presença de matéria orgânica livre (m.o.l) visual

Observa-se, conforme Figura 2A, que o conteúdo de água onde houve a máxima densidade aparente, para o ensaio sem a presença de matéria orgânica livre, variou de 0,245 a $0,254 \mathrm{~g} \mathrm{~g}^{-1}$, com valores máximos de densidade aparente variando de 1,336 a $1,383 \mathrm{~kg} \mathrm{dm}^{-3}$, valores condizentes com a textura do solo estudado.
Para os ensaios do solo com a presença de matéria orgânica livre (Fig. 2B), os valores de conteúdo de água em que ocorreu máxima densidade aparente variaram de 0,273 a $0,286 \mathrm{~g} \mathrm{~g}^{-1}$, enquanto os valores de densidade aparente foram de 1,276 a $1,355 \mathrm{~kg} \mathrm{dm}^{-3}$, com conteúdo de matéria orgânica livre seca visualmente identificada, variando de 2,78 a 9,01 g.

$\mathrm{Na}$ Tabela 3B encontra-se a comparação de média dos valores de conteúdo de água onde a densidade era máxima, valores de densidade máxima alcançada e área sob a curva de compactação, considerando-se a curva de ajuste.

Desta forma e nas condições em que se realizou o ensaio, a matéria orgânica livre não influenciou os valores de densidade aparente, fato que pode ser explicado pelo baixo conteúdo de matéria orgânica livre encontrado em uma das amostras. É interessante salientar que os níveis aqui descritos e, portanto, utilizados no ensaio, foram os encontrados em campo.

Tal estudo não mostrou significância para as variáveis densidade máxima e área sob a curva de compactação, enquanto os valores de conteúdo de água em que a densidade era máxima, mostraram-se significativos em nível de $5 \%$. Encontrou-se conteúdo menor de água no teste sem matéria orgânica livre, sendo que houve maior retenção de água pela matéria orgânica necessitando-se assim, da amostra de um conteúdo de água maior que a amostra sem matéria orgânica livre para um mesmo nível de lubrificação entre as partículas, chegando-se ao mesmo ponto, estatisticamente, de densidade aparente máxima para o ensaio de Proctor. Com base no Cambissolo, deve-se considerar a área específica de contato, e o formato das partículas.

\section{CONCLUSÕES}

1. O Cambissolo estudado apresentou valores elevados de matéria orgânica livre, porém não apresentou diferença estatística para as variáveis analisadas.

2. O Latossolo estudado apresentou valores maiores no conteúdo de água onde a densidade aparente é máxima para o ensaio de Proctor, quando da presença de matéria orgânica livre.

\section{LITERATURA CITADA}

Buckman, H.O. Natureza e propriedades dos solos. Compêndio Universitário sobre edafologia. 4 ed. São Paulo: Freitas Bastos, 1976.

Camargo, O.A.; Alleoni, L.R.F. Compactação do solo e o desenvolvimento das plantas. Piracicaba: SNT, 1997. 132p.

Ekwue, E. I.; Stone, R. J. Organic matter effects on strength properties of compacted agricultural soils. Transactions of the ASAE, St. Joseph, v.38, n.2, p.357-367, 1995.

Figueiredo, L.H.A.; Dias Junior, M. de S.; Santos, G.A.; Bueno Filho, I.S. de S. Efeito dos manejos do solo na densidade do solo máxima e umidade ótima de compactação, usando o ensaio de Proctor normal, em um Latossolo Roxo. In: Congresso Brasileiro de Engenharia Agrícola, 27, 1998, Poços de Caldas. Anais... Lavras: UFLA/SBEA, 1998. v.4, p.103-105. 
Forsythe, W. Manual de laboratorio de fisica de suelos. San Jose: Instituto Interamericano de Ciências Agrícolas, 1980. 212p.

Jorge, J.A. Compactação do solo, causas, conseqüências e maneiras de evitar sua ocorrência. Campinas: Instituto Agronômico de Campinas, 1993, 23p. Circular Técnica 22

Kiehl, E.J. Manual de edafologia. Piracicaba: Editora Ceres, 1979. 262p.

Kondo, M.K.; Dias Junior, M. de S. Efeito da história de tensão e da umidade na compressibilidade de três Latossolos sob diferentes usos. In: Congresso Brasileiro de Engenharia Agrícola, 27, 1998, Poços de Caldas. Anais... Lavras: UFLA/ SBEA, 1998. v.4, p.100-102.

Lanças, K.P.; Upadhyaya, S.K. Pneus radiais para tratores: Guia para seleção correta da pressão de inflação. Energia na Agricultura, Botucatu, v.12, n.1, p.33-36. 1997.

Lasssus, C. Composição dos resíduos vegetais em um solo manejado com nove sistemas de cultivo. Revista Brasileira de Ciência do Solo, Campinas, v.14, n.3, p.375-380, 1990.

Martins Junior, J.X. Métodos de ensaio. Brasília: Divisão de Pesquisas Tecnológicas/Departamento Nacional de Estradas de Rodagem. Ministério da Viação e Obras Públicas. 1964. 206p.

Neujahr,E.B.; Ferreira,M.F.; Schlosser, J.F.; Schneider, V. Comportamento da área de contato e raio dinâmico de pneu agrícola diagonal em função da pressão interna. In: Congresso Brasileiro de Engenharia Agrícola, 27, 1998, Poços de Caldas. Anais... Lavras: UFLA/SBEA, 1998. v.4, p.241-243.
Novak. L.R.; Mantovani, E.C.; Martyn,P.J.; Fernandes, B. Efeito do tráfego de trator e da pressão de contato do pneu/solo na compactação de um Latossolo Vermelho Escuro, Álico, em dois níveis de umidade. Pesquisa Agropecuária Brasileira, Brasília, v.27, n.12, p.1587-1595, 1992.

Oliveira, J.B. De; Jacomine, P.K.T.; Camargo, M.N. Classes gerais de solos do Brasil. Guia para reconhecimento. 2. ed., Jaboticabal: FUNEP, 1992. 201p.

Primavesi, A. Manejo ecológico do solo. A agricultura em regiões tropicais. 9. ed. São Paulo: Livraria Nobel, 1986. 549 p.

Salton, J. C.; Mielniczuk, R. Relações entre sistemas de preparo, temperatura e umidade de um Podzólico Vermelho Escuro de Eldorado do Sul - RS. Revista Brasileira de Ciência do Solo, Campinas, v.19, n.2, 1995.

Schlosser, J.F.; Marquez, L.; Linares, P.; Efeito de diferentes relações cinemáticas entre eixos de um trator agrícola. In: Congresso Brasileiro de Engenharia Agrícola, 27, 1998, Poços de Caldas. Anais... Lavras: UFLA/SBEA, 1998. v.4, p.55-57.

Trein, C. R.; Bergamaschi, H. Influência da compactação pelo pneu do trator no rendimento do milho em plantio direto sob irrigação. In: Encontro Nacional de Plantio Direto na Palha, 6, 1998, Brasília. Resumos... Brasília: FEBRAPDP APDC, 1998.p.72-73. 